\title{
A NOTE ON GRIFFITH CRACKS †
}

\author{
by M. LOWENGRUB
}

(Received 4th April 1966)

1. The problem of determining the distribution of stress in the neighbourhood of a Griffith crack, defined by $|x| \leqq 1, y=0$, which is subject to an internal pressure varying along the length of the crack has been solved by Sneddon and Elliott (3) and by England and Green (1). In the former paper a Fourier cosine method is used to arrive at a solution while in the latter paper the problem is reduced to an Abel integral equation by making use of integral representations of the complex potentials given in Green and Zerna (2). Neither paper deals with the calculation of the stress intensity factor

$$
N=\lim _{x \rightarrow 1^{+}} \sqrt{ }(x-1) \sigma_{y y}(x, 0)
$$

which is extremely important to workers in fracture mechanics.

In this note, simple expressions, equations (12) and (13) below, are derived for $N$ and the energy $W$ required to open up the crack in terms of a general pressure distribution $f(x)$. The solution in (3) is obtained by use of Titchmarsh's solution of the relevant dual integral equations while here we make use of an elementary solution similar to that of Sneddon (4) for the pair of equations arising in the problem of the penny-shaped crack.

2. Due to the symmetry of the problem we need only calculate the distribution of stress in the half space $y \geqq 0$ when the boundary $y=0$ is subject to the conditions $\sigma_{y y}(x, 0)=-f(x), 0<x \leqq 1, \sigma_{x y}(x, 0)=0$ for all $x, u_{y}(x, 0)=0$ $x>1$, and $f(x)=f(-x)$.

It is a simple matter (see pp. $405-406$ of (5)) to show that, if we use the notation

$$
\begin{aligned}
& \mathscr{F}_{c}[f(\xi, y) ; \xi \rightarrow x]=\sqrt{\frac{2}{\pi}} \int_{0}^{\infty} f(\xi, y) \cos (\xi x) d \xi \\
& \mathscr{F}_{s}[f(\xi, y) ; \xi \rightarrow x]=\sqrt{\frac{2}{\pi}} \int_{0}^{\infty} f(\xi, y) \sin (\xi x) d \xi
\end{aligned}
$$

to denote the Fourier cosine and sine transform of $f(\xi, y)$ respectively, a solution of the equations of equilibrium appropriate to the half-plane $y \geqq 0$ is given by

$\dagger$ This research was supported by the United States Air Force under grant AF-AFOSR 435-63, monitored by the Air Force Office of Scientific Research. 
the equations

$$
\begin{aligned}
& u_{x}(x, y)=-\frac{2(1+\eta)}{E} \sqrt{\frac{\pi}{2}} \mathscr{F}_{s}\left[\xi^{-1}(1-2 \eta-\xi y) \psi(\xi) e^{-\xi y} ; \xi \rightarrow x\right] \\
& u_{y}(x, y)=\frac{2(1+\eta)}{E} \sqrt{\frac{\pi}{2}} \mathscr{F}_{c}\left[\xi^{-1} \psi(\xi)(2-2 \eta+\xi y) e^{-\xi y} ; \xi \rightarrow x\right]
\end{aligned}
$$

where $E$ is the Young's modulus and $\eta$ is the Poisson's ratio of the material. We can easily deduce in this case that the stress field is given by

$$
\begin{aligned}
& \sigma_{x x}(x, y)=-\sqrt{\frac{2}{\pi} \mathscr{F}_{c}\left[\psi(\xi)(1-\xi y) e^{-\xi y} ; \xi \rightarrow x\right]} \\
& \sigma_{y y}(x, y)=-\sqrt{\frac{2}{\pi}} \mathscr{F}_{c}\left[\psi(\xi)(1+\xi y) e^{-\xi y} ; \xi \rightarrow x\right]
\end{aligned}
$$

and

$$
\sigma_{x y}=-\sqrt{\frac{\pi}{2}} y \mathscr{F}_{s}\left[\xi \psi(\xi) e^{-\xi y} ; \xi \rightarrow x\right],
$$

so that the boundary conditions are satisfied provided that $\psi(\xi)$ is determined by the pair of equations:

$$
\begin{gathered}
\frac{d}{d x}\left\{\mathscr{F}_{s}\left[\xi^{-1} \psi(\xi) ; x\right]\right\}=\sqrt{\frac{\pi}{2}} f(x), \quad 0 \leqq x<1 \\
\mathscr{F}_{c}\left[\xi^{-1} \psi(\xi) ; x\right]=0, \quad x>1 .
\end{gathered}
$$

The solution of these equations is quite straightforward.

It is given by

$$
\psi(\xi)=\xi \int_{0}^{1} t p(t) J_{0}(\xi t) d t
$$

where

$$
p(t)=\int_{0}^{t} \frac{f(v) d v}{\sqrt{\left(t^{2}-v^{2}\right)}}
$$

3. From equation (2) we find

$$
u_{y}(x, 0)=\frac{2\left(1-\eta^{2}\right)}{E} \sqrt{\frac{\pi}{2}} \mathscr{F}_{c}\left[\xi^{-1} \psi(\xi) ; x\right] .
$$

Using (8) and the result that

$$
\int_{0}^{\infty} J_{0}(\xi t) \cos (\xi x) d \xi=\left(t^{2}-x^{2}\right)^{-\frac{1}{2}} H(t-x)
$$

we have for $0 \leqq x<1$,

$$
u_{y}(x, 0)=\frac{4\left(1-\eta^{2}\right)}{\pi E} \int_{x}^{1} \frac{t p(t) d t}{\sqrt{\left(t^{2}-x^{2}\right)}}
$$


The normal component of stress along the line of the crack is given by equation (4) as:

$$
\sigma_{y y}(x, 0)=-\sqrt{\frac{2}{\pi}} \mathscr{F}_{c}[\psi(\xi) ; x] .
$$

If we represent equation (8) in the form

$$
\psi(\xi)=p(1) J_{1}(\xi)-\int_{0}^{1} t p^{\prime}(t) J_{1}(\xi t) d t
$$

and make use of the fact that

$$
\int_{0}^{\infty} J_{1}(\xi t) \cos (\xi x) d \xi=\frac{1}{t}-\frac{x H(x-t)}{t \sqrt{\left(t^{2}-x^{2}\right)}}
$$

we find that, for $x>1$,

$\sigma_{y y}(x, 0)=\frac{2}{\pi}\left\{\left[x\left(x^{2}-1\right)^{-\frac{1}{2}}-1\right] p(1)+x \int_{0}^{1} \frac{p^{\prime}(t)}{\sqrt{\left(x^{2}-t^{2}\right)}} d t-\int_{0}^{1} p^{\prime}(t) d t\right\}$.

It is clear that if $p(t)$ is differentiable in the neighbourhood of $t=1$,

$$
\int_{0}^{1} \frac{p^{\prime}(t) d t}{\sqrt{ }\left(x^{2}-t^{2}\right)}=O(1) \text { as } x \rightarrow 1^{+}
$$

and thus the stress intensity factor

$$
N=\lim _{x \rightarrow 1^{+}} \sqrt{ }(x-1) \sigma_{y y}(x, 0)
$$

is given by the simple formula

$$
N=\frac{\sqrt{2}}{\pi} p(1)
$$

Substituting from equation (9) we see that

$$
N=\frac{\sqrt{2}}{\pi} \int_{0}^{1} \frac{f(v) d v}{\sqrt{\left(1-v^{2}\right)}}
$$

4. The work done in opening the crack is easily seen to be

$$
W=-2 \int_{0}^{1} \sigma_{y y}(x, 0) u_{y}(x, 0) d x=\frac{8\left(1-\eta^{2}\right)}{\pi E} \int_{0}^{1} f(x) d x \int_{x}^{1} \frac{t p(t) d t}{\sqrt{\left(t^{2}-x^{2}\right)}} .
$$

Interchanging the orders of integration and making use of equation (9) we see that

$$
W=\frac{8\left(1-\eta^{2}\right)}{\pi E} \int_{0}^{1} t[p(t)]^{2} d t
$$

with $p(t)$ given by (9). 
For example, if $f(x)=P_{0}$, a constant, we see that

$$
p(t)=\frac{\pi}{2} P_{0}
$$

yielding

$$
N=\frac{1}{\sqrt{2}} P_{0} \text { and } W=\frac{\pi P_{0}^{2}\left(1-\eta^{2}\right)}{E}
$$

\section{REFERENCES}

(1) A. H. England and A. E. Green, Some two-dimensional punch and crack problems in classical elasticity, Proc. Cambridge Phil. Soc. 59 (1963), 489.

(2) A. E. Green and W. ZERnA, Theoretical Elasticity (Oxford University Press, 1954).

(3) I. N. SNeddon and H. A. ElliotT, The opening of a Griffith crack under internal pressure, Quart. Appl. Math. 4 (1946), 262.

(4) I. N. SNEDdon, The elementary solution of dual integral equations, Proc. Glasgow Math. Assoc. 4 (1960), 108.

(5) I. N. SnEddon, Fourier Transforms (McGraw-Hill; New York, 1951).

Wesleyan AND DUKe UNIVERSITIES 